\title{
Tendencias, patrón de consumo y perfil sociodemográfico de usuarios de heroína en tratamiento
}

\author{
Solveig E. Rodríguez-Kuri', Alma Delia Gutiérrez-López', Marco Antonio Rios-Salinas', Carmen Fernández-Cáceres' \\ 'Centros de Integración Juvenil, A. C.
}

\section{RESUMEN}

Introducción: los opioides son las sustancias responsables del mayor número de muertes por uso de drogas en todo el mundo. Según la Encuesta Nacional de Consumo de Drogas, Alcohol y Tabaco 2016-2017, en México $0.2 \%$ de la población, un poco más de 145 mil personas, ha usado heroína alguna vez en la vida. Objetivo: analizar las tendencias del consumo de heroína, incluyendo la estimación de la tasa media de crecimiento anual en pacientes atendidos en Centros de Integración Juvenil (CIJ) entre 2013 y 2019, así como explorar y comparar su perfil sociodemográfico y patrón de consumo con el de usuarios de otras drogas ilícitas. Método: estudio basado en el análisis de tendencias y en la estimación de la tasa media de crecimiento anual a partir de bases de datos de un registro censal de pacientes, así como en el análisis comparativo basado en pruebas bivariadas de dos grupos de usuarios de drogas. Resultados: la proporción de pacientes consumidores de heroína presentó un incremento gradual hasta mediados del periodo 2013-2019, alcanzando su mayor prevalencia en 2016, para comenzar a caer a partir de ahí, especialmente entre los hombres. El perfil sociodemográfico de los usuarios de heroína revela una mayor afectación física y psicosocial con respecto a los de otras sustancias. Conclusiones: hay una disminución de la demanda de tratamiento, al menos en ClJ, consistente con la prevalencia observadas en población general, aunque dicha disminución no se presenta entre las mujeres, pues principalmente entre las más jóvenes se observa un incremento en el consumo.

Palabras clave: drogas, opiáceos, heroína, tendencias, perfil sociodemográfico, patrón de consumo.

\begin{abstract}
Introduction: opioids are the substance responsible for the highest number of deaths from drug use worldwide. According to the Survey on Drug, Alcohol and Tobacco Use in Mexico, $0.2 \%$ of the population (just over 145,000 people) have used heroin once in their lives. Objective: to analyze the trends in heroin use, including the estimation of the average annual growth rate, in patients treated at $\mathrm{ClJ}$ between 2013 and 2019 and to explore and compare their sociodemographic profile and pattern of use with that of users of other illicit drugs. Method: a study based on the analysis of trends and the estimation of the average annual growth rate from databases of a census registry of patients, as well as on comparative analysis, based on bivariate tests of two groups of drug users. Results: the proportion of heroin-consuming patients showed a gradual increase until the middle of the period 2013-2019, reaching its highest prevalence in 2016, to start falling from there, especially among men. The sociodemographic profile of heroin users shows a greater physical and psychosocial impact compared to those of other substances. Conclusions: there is a decrease in the demand for treatment, at least in ClJ, consistent with the prevalence observed in the general population, highlighting that this decrease does not occur among women, especially among younger women, even an increase in consumption is observed.
\end{abstract}

Keywords: drug abuse, opiates, heroine, trends, profile of drug users, drug abuse pattern.

\footnotetext{
Autor de correspondencia:

Marco Antonio Rios Salinas. Av. San Jerónimo núm. 372, col. Jardines de Pedregal, alcaldía Álvaro Obregón, Ciudad de México, C.P. 01900. Correo electrónico: marco.rios@cij.gob.mx

Recibido: 15 de abril de 2020

Aceptado: 14 de mayo de 2020

doi: 10.28931/riiad.2020.1.04
} 


\section{INTRODUCCIÓN}

Los opioides son las drogas responsables del mayor número de muertes en personas con trastorno por uso de sustancias en el mundo (UNODC, 2019; Observatorio Europeo de las Drogas y las Toxicomanías y Europol, 2016). De acuerdo con el último informe de la Oficina de las Naciones Unidas contra las Drogas y el Delito (UNODC, 2019), en 2017 poco más de 53 millones de personas en el mundo habían consumido opioides durante el año anterior. De estos, casi 30 millones habían utilizado opiáceos como la heroína y el opio, lo que representa un incremento del 56\% respecto a 2016, ello también explica la implementación de nuevas encuestas en países con alta concentración de población, como la India.

En México, según la Encuesta Nacional de Consumo de Drogas, Alcohol y Tabaco 2016-2017 (INPRFM, INSP, CONADIC, SS, 2017), poco más de 145 mil personas, 0.2\% de la población del país, entre 12 y 65 años de edad, ha consumido heroína alguna vez (AV) en la vida. Se trata, en su gran mayoría, de hombres, los cuales suman poco más de 140 mil.

El rango de edad en el que se concentra la mayor cantidad de usuarios alguna vez en la vida (AV) es de 18 a 34 años de edad; sin embargo, la población masculina con mayor prevalencia de consumo se concentra en los rangos de 18 a 34 años y de 35 a 65 años, mientras que la población de mujeres con mayor prevalencia se encuentra entre los 12 y 17 años y los 18 y 34 .

Una comparación con los datos obtenidos en la Encuesta Nacional de Adicciones de 2011 (SS, CONADIC, INPRFM, INSP, 2011) muestra que la prevalencia de consumo de heroína AV se mantuvo estable durante un periodo de cinco años, en el cual se observa incluso una disminución en términos absolutos, de 178 mil consumidores en 2011 a 145 mil en 2016, principalmente entre la población masculina. $Y$ aunque el consumo durante el último año (UA) muestra un incremento de casi el doble, pasando de 10 mil a más de 23 mil usuarios, la prevalencia AV, que es acumulativa, pareciera indicar que no se han incorporado cantidades importantes de nuevos usuarios.

Por su parte, entre la población de estudiantes, se observan prevalencias bastante mayores que en la población general (INPRFM, CONADIC, SS, 2015). Es así que 0.9\% de los estudiantes de educación media y media superior ha consumido heroína alguna vez en la vida (AV), 0.5\% lo ha hecho en el último año (UA) y $0.3 \%$ en el último mes (UM). Lo más preocupante de los datos arrojados por esta encuesta es que los alumnos de secundaria presentan prevalencias ligeramente mayores que los de bachillerato y que las diferencias de la prevalencia por sexo son mucho menores que las observadas en la población general, debido a un mayor consumo de esta sustancia por parte de las jóvenes.
Lo anterior cobra mayor relevancia si se considera que los usuarios de heroína constituyen una población especialmente de riesgo, debido a que, como se encuentra documentado, en comparación con otros grupos de usuarios, suelen desarrollar patrones de consumo más severos y presentan una mayor afectación física y social (Baldwin et al., 2018; Berbesi, Segura-Cardona, Montoya-Vélez, \& Mateu-Gelabert, 2013; Berbesi-Fernández, Segura-Cardona, Montoya-Velez, \& Ramirez Medina, 2016; Cravioto, Medina-Mora, de la Rosa, Galván, \& Tapia-Conyer, 2003; De Molina et al., 2007; Gutiérrez et al., 2001; Isaza, Suárez, Henao, \& González, 2010; Jain, \& Fisher, 2018; Meacham et al., 2015; Parker, Egginton, \& Elson, 2001; Rodríguez et al., 2005; Romo-González, \& Larralde, 2013; Sanvisens et al., 2014; Schilling, 2019.)

¿De qué manera el comportamiento observado en estas encuestas se puede ver reflejado en la demanda de tratamiento? Para responder esto y con el objeto de conocer con mayor profundidad las características de la demanda de tratamiento, es decir, su evolución en el tiempo, el perfil sociodemográfico de los usuarios, su patrón de consumo, los principales problemas asociados al mismo y para determinar si dicha población constituye un grupo claramente diferenciado y con necesidades de tratamiento y rehabilitación específicas respecto a otros grupos de usuarios, se llevó a cabo el presente estudio con base en los siguientes dos objetivos.

El primer objetivo consistió en analizar las tendencias y estimar la tasa media de crecimiento del consumo de heroína en solicitantes de tratamiento de CIJ durante el periodo de 2013 a 2019, tomando en cuenta a la población total y a la población por sexo. Este periodo se estableció considerando el primer año en el que se comenzó a tener registro de la vía o forma de administración de las sustancias de consumo, a fin de posibilitar la agrupación por separado de los consumidores de heroína por vía intravenosa y descartar de inicio que pudieran constituir una población diferente de los demás respecto a las variables de interés para este estudio. Sin embargo, debido a que no se observaron diferencias significativas entre los usuarios con distintas formas de administración, se optó por integrar en un mismo grupo a todos los usuarios de heroína.

El segundo objetivo del estudio consistió en explorar las características sociodemográficas, el patrón y tipo de consumo y los problemas asociados al mismo de los usuarios de heroína atendidos durante 2019 y compararlos con los de los usuarios de otras drogas ilícitas, con el fin de identificar diferencias estadísticamente significativas que permitieran considerar a los usuarios de heroína como un grupo claramente diferenciado de los de otras drogas (los indicadores de cada una de estas variables se describen en el apartado metodológico). 


\section{MÉTODO}

Para arribar al primer objetivo se utilizó un diseño de tendencia o trend que permite analizar cambios en una población dentro de un periodo con mediciones en distintos puntos de ese lapso.

La información procede de bases de datos del Sistema de Información Epidemiológica del Consumo de Drogas (SIECD) de CIJ $^{1}$ entre los años 2013 y 2019, el cual reúne y valida cada seis meses datos censales obtenidos mediante un reporte del propio paciente en una entrevista inicial asimilada a un expediente electrónico, realizada por personal capacitado en el tratamiento médico o psicoterapéutico de adicciones, acerca de sus características sociodemográficas, determinantes y motivos de consulta, problemas asociados y patrón de consumo, esto último con base en el registro de un grupo de 26 sustancias. Entre las variables exploradas en este rubro se incluyen: uso alguna vez en la vida, en el último año y en el último mes, edad de inicio del consumo, drogas de inicio, tiempo trascurrido antes de solicitar tratamiento, drogas de mayor impacto y episodios de abuso en los últimos 30 días.

Para efectos de este estudio, se retomó la información sobre prevalencias de consumo AV, UA, UM y sexo.

En la primera parte del estudio se incluyeron los datos de un total de 159,981 personas que asistieron a solicitar tratamiento entre 2013 y 2019 (130,732 hombres y 29,248 mujeres).

La segunda parte consistió en un análisis comparativo entre usuarios de heroína y usuarios de otras drogas que ingresaron a tratamiento durante 2019. La población de estudio se conformó por 22,834 usuarios de drogas ilícitas. El primer grupo se conformó por 550 usuarios de heroína AV, en tanto que el segundo incluyó al resto de los usuarios que reportaron consumo de cualquier otra droga ilegal distinta de la heroína, esto es 22,284 pacientes.

Para ello se reunió información sobre datos sociodemográficos, problemas asociados y patrón de consumo en sus tres modalidades (AV, UA, UM) de un conjunto de sustancias, entre las que se incluyen mariguana, tabaco, alcohol, cocaína, inhalantes, metanfetaminas, éxtasis, benzodiacepinas, alucinógenos y heroína, así como edad de inicio y lapso transcurrido previo a la solicitud de tratamiento.

\section{Análisis}

En la primera parte se incluye un análisis descriptivo de las tendencias observables entre 2013 y 2019 a nivel nacional en hombres y mujeres, tanto para el consumo AV,
UA y UM. Posteriormente, se calculó la tasa media de crecimiento anual real (TMCAR) de consumidores de heroína entre 2013 y 2019, controlando la tasa de crecimiento anual de los ingresos a tratamiento en dicho periodo. La estimación de la tasa de crecimiento anual se calculó mediante la siguiente fórmula: $(\mathrm{N} / \mathrm{n})^{\wedge}(1 / 6)-1$, donde $\mathrm{N}$ es la población total en 2013; n es la población total en 2019 y 6 se refiere a los años transcurridos entre 2013 y 2019.

Para la segunda parte se usaron pruebas no paramétricas, como Ji Cuadrada (x2), y paramétricas, como $t$ de Student $(t)$, según fuese el caso, para comparar el patrón de consumo y demás variables antes mencionadas entre el grupo de usuarios de heroína y el de otras drogas. En el caso de la $t$ de Student se utilizó la prueba de Leveane para determinar la igualdad de varianzas entre las muestras. Los análisis se realizaron mediante el programa SPSS v. 19.

\section{Consideraciones éticas}

Para este estudio se emplearon bases de datos con información proporcionada por usuarios de drogas que solicitaron tratamiento en Centros de Integración Juvenil durante una entrevista inicial que forma parte de los procedimientos institucionales de ingreso a tratamiento, momento en el cual se les comunica que la información proporcionada puede ser utilizada con fines estadísticos y de investigación; en todo momento se respeta el anonimato y la confidencialidad de la misma y se solicita su consentimiento para estos fines.

\section{RESULTADOS}

De las 159,981 personas que solicitaron atención por uso de drogas en Centros de Integración Juvenil entre los años 2013 y 2019, 4,804 refirieron haber consumido heroína alguna vez en la vida (AV), lo que representa 3.0\% del total de usuarios de primer ingreso a tratamiento; un porcentaje menor (2.2\%) reportó haber consumido heroína en el último año (UA) y $1.4 \%$ lo hizo durante el último mes (UM). Cabe señalar que 130,732 eran hombres $(81.7 \%)$ y 29,248 eran mujeres (18.3\%).

\section{Tendencias del consumo de heroína}

Como se observa en la Figura 1, la proporción de usuarios de heroína (AV, UA y UM) que solicitó tratamiento en ClJ presentó un decremento en el periodo 2013-2019, alcanzando su prevalencia máxima en 2016.

\footnotetext{
${ }^{1}$ Organización especializada en la prevención, tratamiento e investigación del consumo de drogas, con 117 unidades de atención distribuidas en todo el país.
} 
Figura 1. Tendencias del consumo de heroína en usuarios de drogas de primer ingreso a tratamiento a ClJ. 2013-2019

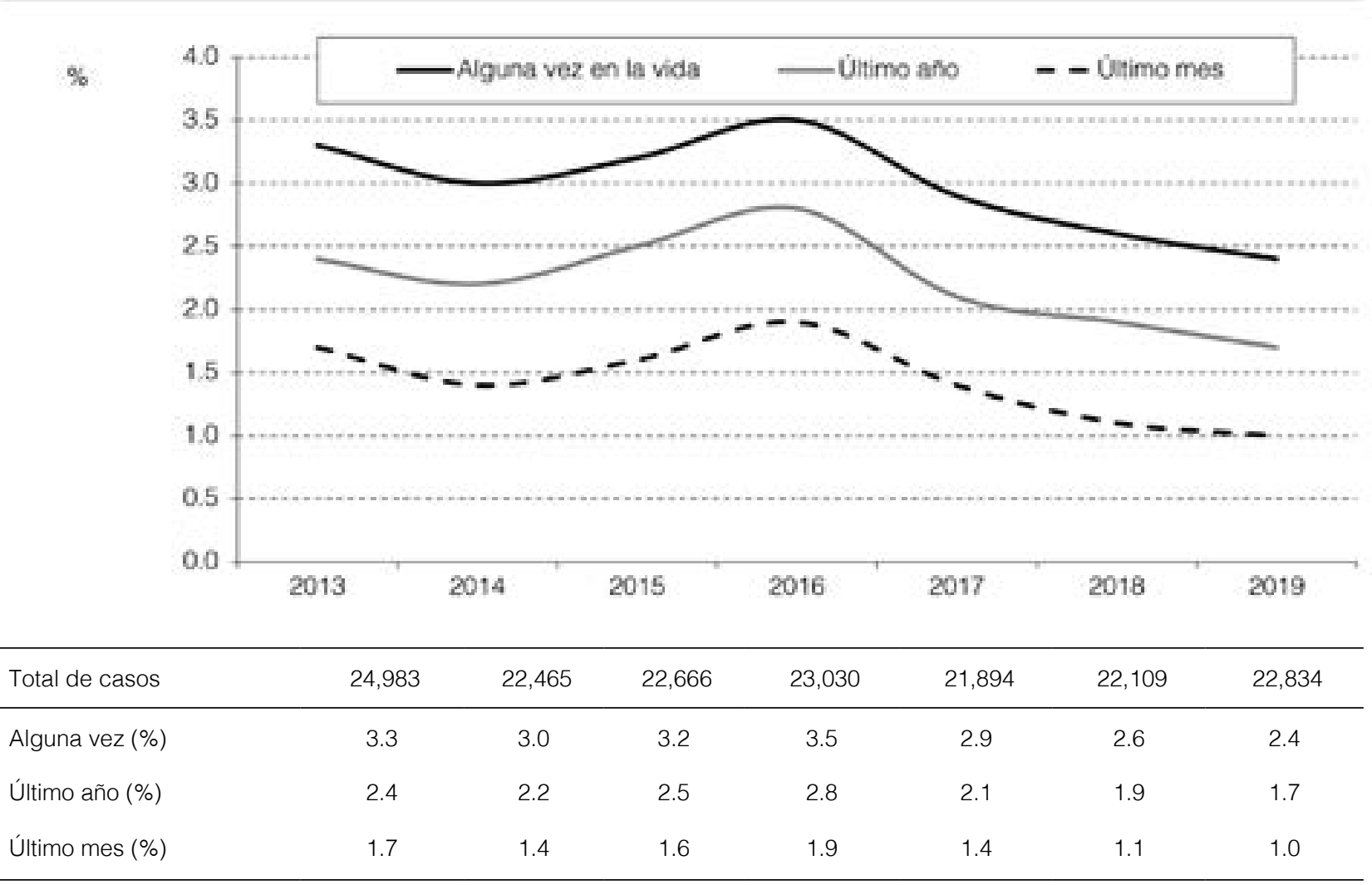

Una comparación entre hombres y mujeres (ver Figura 2) permite observar que, en general, el consumo de heroína en los hombres es superior al registrado por las mujeres. Sin embargo, mientras que los hombres registran una disminución del consumo en las tres modalidades (AV, UA y $U M)$, las mujeres presentan un incremento en las modalidades AV y UA, y únicamente disminuyen su consumo en el UM, de modo que desde 2017 las prevalencias entre ambos sexos comienzan a igualarse.

La estimación de la tasa media de crecimiento anual (TMCAR) muestra claramente este comportamiento. Como se observa en la Figura 3, dicho crecimiento es negativo en las tres modalidades de consumo de esta sustancia (AV, UA y UM) para el total de la población y para los hombres en particular. En tanto que en el caso de las mujeres destaca, por un lado, el incremento en el consumo AV y UA, y por otro, una disminución en el del UM.

\section{Comparación del perfil sociodemográfico, problemas asociados y patrón de consumo entre usuarios de heroína y de otras drogas}

Entre los pacientes consumidores de heroína, la proporción hombres-mujeres es de cuatro a uno aproximada- mente, semejante a la de los usuarios de otras drogas (ver Tabla 1), lo que no ocurre con respecto a la edad de ingreso a tratamiento, que es de 32.4 años en promedio para los usuarios de heroína $(D E=12.2)$, contra 23.8 años $(D E=10.7)$ para los usuarios de otras drogas $(t=$ 16.468, $g l=570.061, p<.001)$.

Quizás debido a su mayor edad, entre los usuarios de heroína se observa un porcentaje más amplio de pacientes que ha estado casado o que vive o ha vivido en pareja en comparación con los usuarios de otras drogas ( $50.2 \%$ contra 29.7\%; $x 2=106.952, g l=1, p<.001)$. De los usuarios de heroína que han vivido en pareja, $17.1 \%$ se encuentra hoy en día separado, divorciado o viudo, contra $7.7 \%$ de los usuarios de otras drogas que ha tenido una separación de este tipo ( $x 2=64.522, g l=1, p<.001)$, lo que puede sugerir una mayor afectación de la vida familiar en el caso de los usuarios de heroína.

Por otra parte, se observa un nivel de estudios significativamente menor entre los usuarios de heroína. La proporción de usuarios de heroína que no cuenta con estudios o no sabe leer o escribir es de $1.6 \%$, en comparación con $0.5 \%$ de los usuarios de otras drogas en la misma situación ( $x 2=11.507, g /=1, p=.001$ ), y entre quienes sólo cuentan con primaria la proporción es de 
Figura 2. Tendencias del consumo de heroína en el último año según sexo en usuarios de drogas de primer ingreso a tratamiento a CIJ. 2013-2019

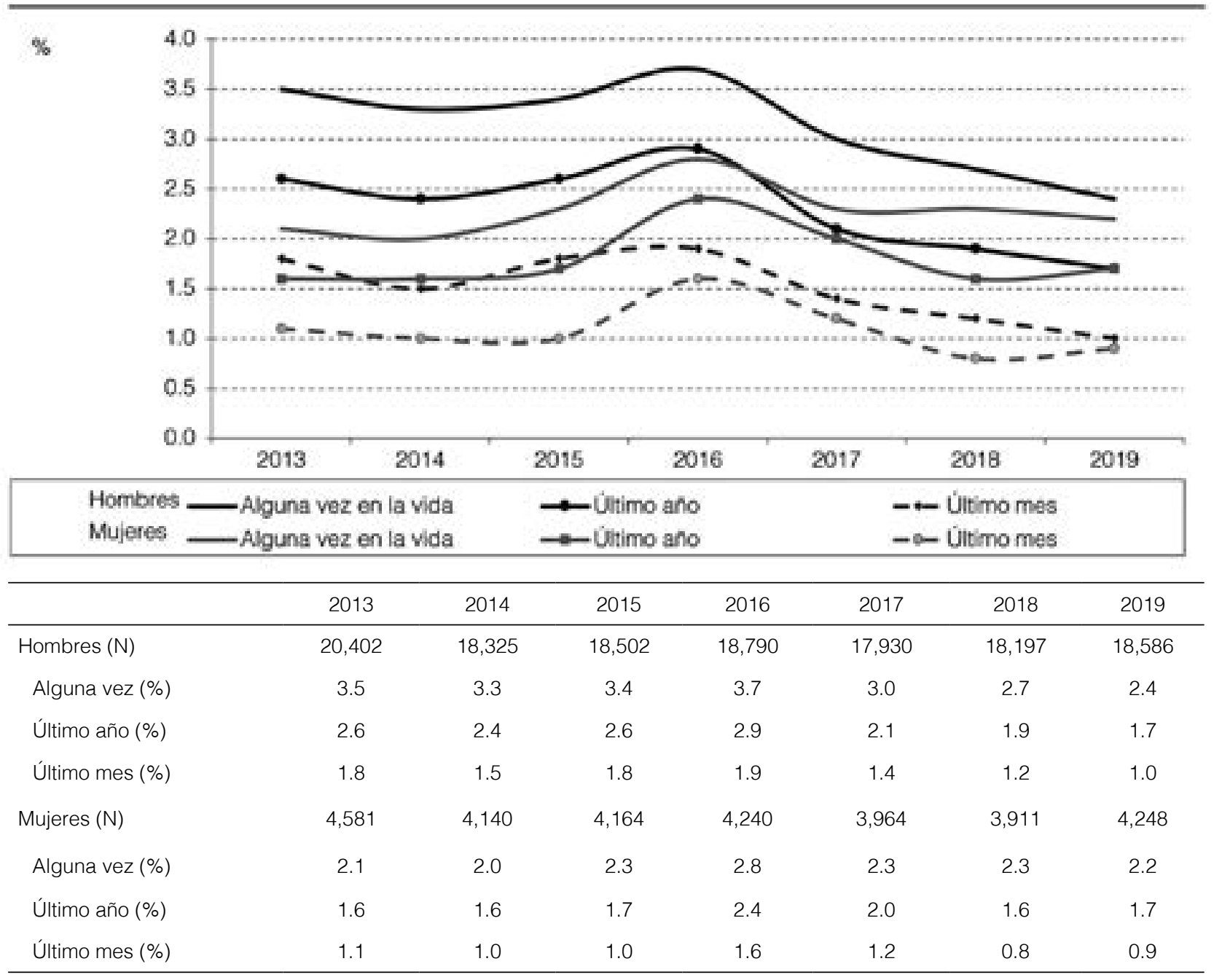

$14.5 \%$ para usuarios de heroína, en comparación con $6.4 \%$ de quienes consumen otras drogas ( $x 2=58.26$, $g l=1, p<.001)$. En personas con mayor escolaridad sucede lo contrario, los usuarios de heroína con estudios de bachillerato son menos en comparación con los de otras drogas (31.8\% contra $40.2 \%$, respectivamente; $x 2$ $=15.703, g l=1, p<.001)$.

Resalta también la mayor cantidad de desempleados entre los usuarios de heroína, quienes conforman $36.7 \%$ en comparación con los de otras drogas, que suman $21.3 \%(x 2=75.506, g l=1, p<.001)$. El alto porcentaje de desempleados entre los consumidores de heroína, así como las condiciones de vida y socioeconómicas a las que se ha hecho referencia parecen reflejar un mayor nivel de marginación social, lo que resultaría consistente con un tipo de consumo más disfuncional, reportado así por $49.9 \%$ de estos usuarios, en comparación con $22.5 \%$ de los usuarios de otras sustancias que reportan consumo disfuncional ( $\times 2=$ 226.381, $g l=1, p<.001)$.

Aunado a lo anterior, y como se observa en la Figura 4 , se aprecia un mayor deterioro físico de los consumidores de heroína, ya que $69.3 \%$ presenta problemas de salud asociados a su consumo, comparado con $45.3 \%$ de usuarios de otras drogas que reportan problemas en este terreno ( $x 2=123.973, g l=1, p<.001)$. En general, se observan mayores problemáticas en distintos ámbitos que se asocian al consumo de heroína que al de otras drogas, tales como: familiar (85.8\% contra $78.5 \%$; $x 2$ $=16.986, g l=1, p<.001)$, psicológico $(60.9 \%$ contra $45.1 \% ; x 2=54.041, g l=1, p<.001)$, laboral $(50.4 \%$ contra $25.7 \% ; \times 2=168.233, g l=1 p<.001)$, legal (43.8\% contra 18.5\%; $x 2=222.290, g l=1, p<.001)$, conducta antisocial o delictiva (38.4\% contra $13.7 \%$; $x 2$ 
Figura 3. Tasa media de crecimiento anual del consumo de heroína en usuarios de drogas de primer ingreso a tratamiento a ClJ. De 2013 a 2019

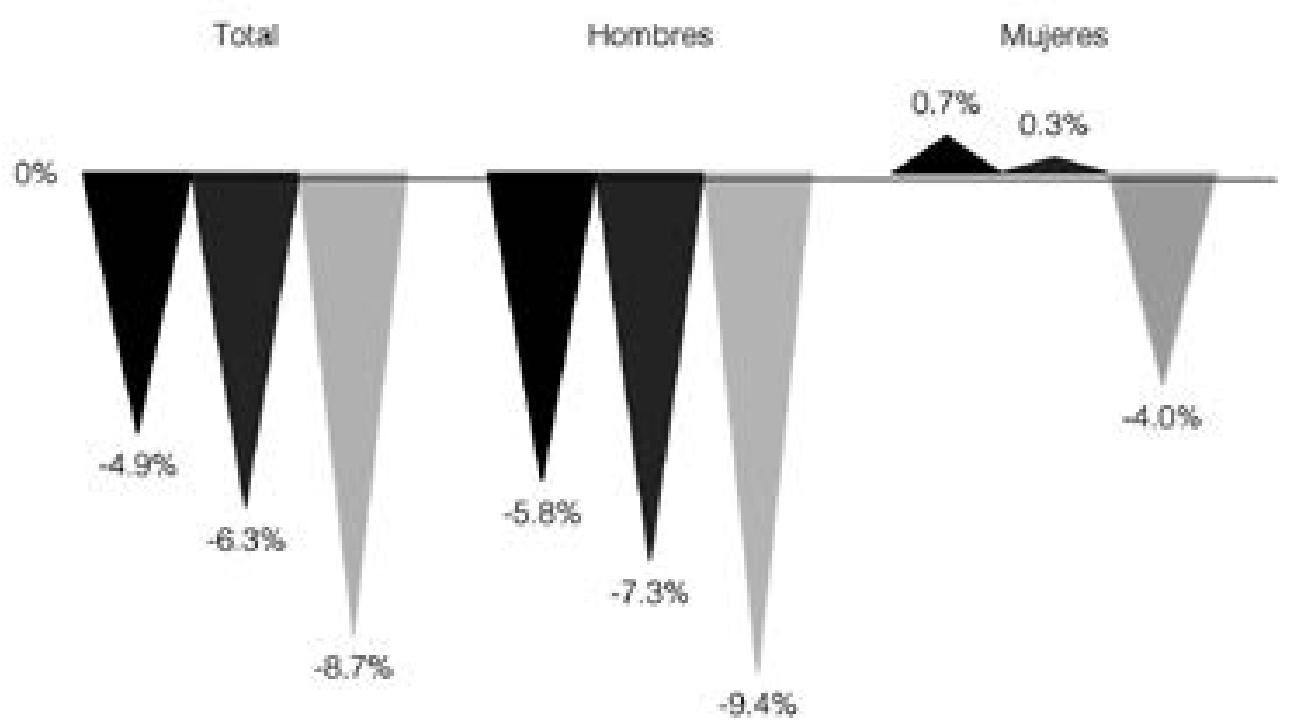

-Alguna vez en la vida aÚltimo añoúltimo mes

Tabla 1

Características sociodemográficas de los usuarios de heroína y de los usuarios de otras drogas de primer ingreso a tratamiento a ClJ en 2019

$\begin{array}{cl}\text { Usuarios de heroína } & \begin{array}{c}\text { Usuarios de } \\ \text { otras drogas } \\ (N=550)\end{array} \\ (N=22227) & X 2(g l)\end{array}$

\begin{tabular}{|c|c|c|c|c|}
\hline \multicolumn{5}{|l|}{ Sexo } \\
\hline Hombres & 82.7 & 81.4 & $0.659(1)$ & 0.417 \\
\hline Mujeres & 17.3 & 18.6 & & \\
\hline \multicolumn{5}{|l|}{ Estado civil } \\
\hline Nunca ha vivido en pareja & 49.8 & 70.3 & & \\
\hline Ha vivido en pareja & 50.2 & 29.7 & $106.952(1)$ & $<.001$ \\
\hline Separado, divorciado y viudo & 17.1 & 7.7 & & \\
\hline Soltero o vive en pareja & 82.9 & 92.3 & $64.522(1)$ & $<.001$ \\
\hline \multicolumn{5}{|l|}{ Escolaridad } \\
\hline Sin estudios & 1.6 & 0.5 & $11.507(1)$ & .001 \\
\hline Primaria & 14.5 & 6.4 & $58.260(1)$ & $<.001$ \\
\hline Secundaria & 38.9 & 37.6 & $0.366(1)$ & .545 \\
\hline
\end{tabular}


Características sociodemográficas de los usuarios de heroína y de los usuarios de otras drogas de primer ingreso a tratamiento a ClJ en 2019 (continuación)

\begin{tabular}{|c|c|c|c|c|}
\hline Estudios técnicos & 2.5 & 2.4 & $0.083(1)$ & .774 \\
\hline Bachillerato & 31.8 & 40.2 & $15.703(1)$ & $<.001$ \\
\hline Estudios superiores & 9.6 & 12.4 & $3.802(1)$ & .051 \\
\hline Estudios de posgrado & 0.9 & 0.5 & $2.021(1)$ & .155 \\
\hline \multicolumn{5}{|l|}{ Ocupación } \\
\hline Desempleado(a) & 36.7 & 21.3 & $75.506(1)$ & $<.001$ \\
\hline Estudiante & 6.9 & 31.7 & $154.197(1)$ & $<.001$ \\
\hline Estudiante que trabaja & 2.5 & 6.0 & $11.606(1)$ & .001 \\
\hline Trabaja & 48.9 & 36.9 & $32.915(1)$ & $<.001$ \\
\hline Tareas del hogar & 4.4 & 3.5 & $1.329(1)$ & .249 \\
\hline Pensionado & 0.5 & 0.6 & $0.008(1)$ & .929 \\
\hline
\end{tabular}

Figura 4. Problemas asociados al consumo de drogas ilícitas en los usuarios de heroína y en los usuarios de otras drogas de primer ingreso a tratamiento a ClJ en 2019

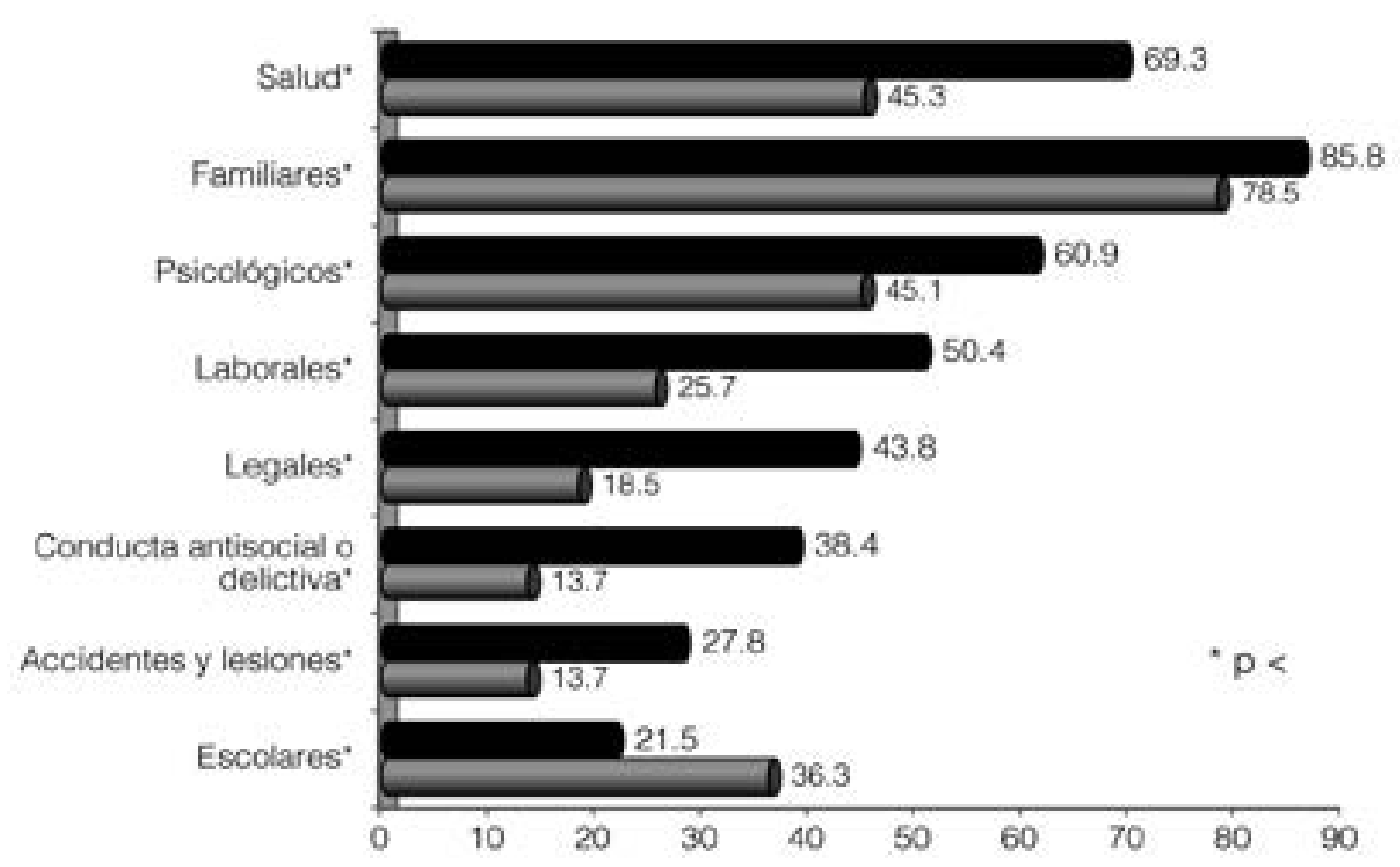

\section{- Usuarios de heroina aUsuarios de otras drogas ilicitas}


$=268.321, g l=1, p<.001)$, presencia de accidentes 0 lesiones (27.8\% contra 13.7\%; $\times 2=89.518, g l=1, p<$ $.001)$ y escolar $(21.5 \%$ contra $36.3 \%$; $x 2=51.178, \mathrm{gl}=$ $1, p<.001$.

De acuerdo con los datos obtenidos, los usuarios de heroína inician el consumo de drogas ilegales a una edad más temprana que los de otras drogas $(M=14.7$; $D E=4.2$ para los primeros y $M=16.4, D E=5.4$ para los segundos; $t=-9.411, g l=594.837, p<.001$ ), a esto hay que agregar los más de seis años que transcurren en promedio antes de que inicien el consumo de heroína $(M=21.2$ años, $D E=6.6)$ y todavía pasarán más de 11 años $(M=11.2, D E=10.8)$ para que asistan a solicitar tratamiento por dicho consumo. Es decir, que al ingresar a tratamiento estos pacientes no sólo tienen casi 12 años de consumir heroína, sino que tienen en promedio 17.7 años $(D E=11.8)$ consumiendo drogas ilegales, contra 7.4 años de los de otras drogas ( $D E=8.9$ ), lo que se refleja en un deterioro psicosocial y físico mayor y en un patrón de consumo más severo. Esto último se observa con especial claridad en la proporción de pacientes usuarios de heroína (60.9\%) que presenta un uso combinado de sustancias (contra $46.1 \%$ de los de otras drogas; $\times 2=$ 42.262, $g l=1, p<.001)$, así como en el número de drogas consumidas a lo largo de la vida $(t=26.872, g l=562.550$, $p<.001)$. En este sentido, como se muestra en la Tabla 2, una proporción significativamente mayor de usuarios de heroína presenta consumo de otras sustancias ilegales, particularmente de benzodiacepinas, metanfetaminas y cocaína en polvo.

Tabla 2

Consumo de sustancias en los usuarios de heroína y en los usuarios de otras drogas de primer ingreso a tratamiento a ClJ en 2019

\begin{tabular}{|c|c|c|c|c|}
\hline & $\begin{array}{l}\text { Usuarios de heroína } \\
\qquad(N=550)\end{array}$ & $\begin{array}{l}\text { Usuarios de } \\
\text { otras drogas } \\
(N=22227)\end{array}$ & $X 2(g l)$ & $p$ \\
\hline \multicolumn{5}{|l|}{ Consumo alguna vez en la vida } \\
\hline Tabaco & 93.8 & 85.0 & $33.210(1)$ & $<.001$ \\
\hline Alcohol & 90.0 & 88.5 & $1.247(1)$ & .264 \\
\hline Mariguana & 91.3 & 86.5 & $10.676(1)$ & .001 \\
\hline Inhalables & 39.3 & 20.8 & $108.713(1)$ & $<.001$ \\
\hline Cocaína en polvo & 76.9 & 40.7 & $289.209(1)$ & $<.001$ \\
\hline Crack & 41.3 & 19.7 & $155.115(1)$ & $<.001$ \\
\hline Metanfetaminas & 71.8 & 36.3 & $290.960(1)$ & $<.001$ \\
\hline Éxtasis y otras metanfetaminas alucinógenas & 18.5 & 6.3 & $130.641(1)$ & $<.001$ \\
\hline Benzodiacepinas & 48.0 & 14.6 & $458.213(1)$ & $<.001$ \\
\hline Alucinógenos & 32.9 & 13.6 & $164.449(1)$ & $<.001$ \\
\hline \multicolumn{5}{|l|}{ Consumidas en el último año } \\
\hline Tabaco & 85.1 & 75.9 & $25.126(1)$ & $<.001$ \\
\hline Alcohol & 77.8 & 81.4 & $4.469(1)$ & .035 \\
\hline Mariguana & 72.0 & 72.2 & $.009(1)$ & .923 \\
\hline Inhalables & 20.9 & 13.4 & $25.563(1)$ & $<.001$ \\
\hline Cocaína en polvo & 49.1 & 27.4 & $125.661(1)$ & $<.001$ \\
\hline Crack & 26.7 & 14.4 & $65.449(1)$ & $<.001$ \\
\hline Metanfetaminas & 59.8 & 32.2 & $185.008(1)$ & $<.001$ \\
\hline Éxtasis y otras metanfetaminas alucinógenas & 9.6 & 3.8 & $47.157(1)$ & $<.001$ \\
\hline Benzodiacepinas & 34.0 & 10.3 & $312.362(1)$ & $<.001$ \\
\hline
\end{tabular}


Consumo de sustancias en los usuarios de heroína y en los usuarios de otras drogas de primer ingreso a tratamiento a ClJ en 2019 (continuación)

\begin{tabular}{lcccc}
\hline & $\begin{array}{c}\text { Usuarios de heroína } \\
(N=550)\end{array}$ & $\begin{array}{c}\text { Usuarios de } \\
\text { otras drogas } \\
(N=22 \text { 227) }\end{array}$ & X2 (gl) & $p$ \\
\hline Alucinógenos & 17.8 & 8.9 & $51.702(1)$ & $<.001$ \\
Consumidas en el último mes & & & & \\
Tabaco & 75.3 & 59.8 & $53.732(1)$ & $<.001$ \\
Alcohol & 50.4 & 56.3 & $7.814(1)$ & .005 \\
Mariguana & 45.3 & 48.3 & $1.969(1)$ & .161 \\
Inhalables & 6.2 & 5.6 & $.310(1)$ & .578 \\
Cocaína en polvo & 12.5 & 9.8 & $4.664(1)$ & .031 \\
Crack & 9.5 & 7.2 & $4.100(1)$ & .043 \\
Metanfetaminas & 33.6 & 20.1 & $60.514(1)$ & $<.001$ \\
Éxtasis y otras metanfetaminas alucinógenas & 1.1 & 0.8 & $.423(1)$ & .516 \\
Benzodiacepinas & 15.3 & 4.7 & $128.565(1)$ & $<.001$ \\
Alucinógenos & 1.8 & 1.8 & $.002(1)$ & .964 \\
\hline
\end{tabular}

\section{CONCLUSIONES}

Se puede afirmar que la demanda de tratamiento por uso de heroína ha mostrado una tendencia a la baja en los últimos seis años, al menos entre pacientes que asisten a solicitar tratamiento a CIJ (Gutiérrez, 2013; Gutiérrez, 2019), lo cual resulta consistente con los datos observados entre la población en general (según las encuestas, poblacionales antes referidas), donde la prevalencia de consumo alguna vez prácticamente no creció en el periodo de 2011 a 2016, e incluso en términos absolutos mostró un ligero decremento, a pesar de que en el último año se observó un aumento entre los hombres adultos (INPRFM et al.,2017). Lo más destacable de estas tendencias es que entre mujeres jóvenes no se registró una disminución en la prevalencia de consumo, esto se puede apreciar tanto en las encuestas con población abierta como en población de estudiantes (INPRFM et al., 2015; INPRFM et al., 2017) y también entre las mujeres que asistieron a tratamiento a $\mathrm{ClJ}$ durante esos años (Gutiérrez, 2013; Gutiérrez, 2019).

Aún es difícil conocer si las razones de esta estabilidad o reducción en las prevalencias y en la demanda de tratamiento en ClJ obedecen a cambios en los patrones de consumo que impliquen la sustitución de esta sustancia por otras; sin embargo, los datos de ClJ muestran que del total de pacientes que reportan consumo de opiáceos, la proporción de consumidores de heroína ha disminuido gradualmente con respecto a otras variedades de ese grupo, de modo que el consumo de opioides sintéticos (entre los que se encuentran medicamentos como fentanilo, darvón, etcétera) registró un importante incremento, al pasar de $0.4 \%$ en el primer semestre de 2013, a 1.3\% en los primeros seis meses de 2019. (Gutiérrez 2013, Gutiérrez, 2019) no obstante, el sistema de registro no permite determinar con precisión el tipo de opioides que está marcando dicho aumento.

También se debe considerar que la oferta de organismos o instituciones dirigidas a la atención de consumidores de heroína en la región más afectada por este problema ha crecido significativamente en los últimos años, por lo que la demanda de tratamiento por uso de esta sustancia se encuentra menos concentrada en las unidades de ClJ.

Por otra parte, aunque se registra una tendencia a la estabilidad en las prevalencias de consumo entre la población en general, no debe perderse de vista el incremento que sí ha ocurrido entre la población más joven, que si bien no ha sido de magnitud suficiente como para impactar la prevalencia total, no deja de prenderse un foco de alerta al observar que los estudiantes de secundaria consumen heroína en mayor proporción que los de bachillerato y que un alto porcentaje de mujeres consumidoras se encuentra en el rango de 12 a 17 años de edad, sólo un poco por debajo del de 18 a 34 años. 
En cuanto a los hallazgos del análisis comparativo, se corroboran los resultados de otros estudios que muestran patrones de consumo más severos y disfuncionales entre usuarios de heroína (Cravioto et al., 2003), una edad de inicio más temprana (Isaza et al., 2010), más daños a la salud (Romo-González, \& Larralde, 2013) y una mayor afectación de la vida social y productiva que se expresa en un menor nivel de estudios, en problemas para mantener relaciones de pareja estables y en un mayor desempleo (De Molina et al., 2007; Gutiérrez et al., 2001; Isaza et al., 2010; Parker et al., 2001), todo lo cual se traduce en un nivel de marginación más profundo que el que se puede observar entre usuarios de otras drogas ilegales. Asimismo, los usuarios de heroína tienden a ser poliusuarios con más frecuencia que los consumidores de otras drogas (Berbesi et al., 2013; Rodríguez et al., 2005). Además, quienes usan heroína a la vez que otras sustancias, en particular cocaína, tienen más alta probabilidad de presentar conductas de riesgo relacionadas con la aplicación de jeringas y con prácticas sexuales, aumentando con ello el riesgo de infecciones de transmisión sexual y la presencia de VIH (Berbesi-Fernández et al., 2016; Cravioto et al., 2003; Meacham et al., 2015)

Además, como se ha documentado ampliamente, la heroína no sólo es una de las drogas que mayor afectación física y psicosocial ocasiona en quienes la consumen, sino que también es una de las que producen una dependencia orgánica más rápida y severa (Brown, 2004; Forsyth, Biggar, Chen, \& Burstein, 2017; Hosztafi, 2011).

En conclusión, se deben priorizar los esfuerzos por favorecer el ingreso más temprano a tratamiento de estos usuarios, no sólo por el alto nivel de deterioro que provoca de por sí la heroína y el elevado riesgo de muerte por sobredosis y daños asociados (Baldwin et al., 2018; Jain \& Fisher, 2018; Schilling, 2019), sino también por el amplísimo lapso que transcurre entre el inicio del uso de esta sustancia y de otras drogas consumidas previamente, y el ingreso del paciente a tratamiento, periodo que algunos autores estiman en 12 años (Sanvisens et al., 2014) pero que, como se ha constatado con pacientes de ClJ, puede llegar a ser hasta de ocho y 10 años.

Conviene prestar atención también, y esto podría constituir un futuro tema de estudio, a la creciente evidencia del uso ilícito de fentanilo a menudo combinado con heroína, en países como Estados Unidos y Canadá, lo que ya constituye una importante causa de muertes por sobredosis en esos países (Baldwin et al., 2018) y podría comenzar a presentarse con mayor frecuencia en México, por lo que habrá que estar preparado para brindar atención a las nuevas tendencias del consumo de opioides, particularmente en las entidades del país que tradicionalmente han registrado consumo de heroína y que en la actualidad comienzan a reportar algunos casos de consumo de fentanilo.

Finalmente, es importante señalar la necesidad de considerar los resultados del presente estudio a la luz de algunas limitaciones. La más importante relacionada con el nivel de representatividad del SIECD, el cual está limitado a la población que asiste a solicitar tratamiento a ClJ. No obstante, esta información resulta útil para identificar los factores de riesgo relativos al sexo de los pacientes, problemas asociados y necesidades de rehabilitación, así como tendencias para la planeación de programas de tratamiento e indicadores para su evaluación. También deben tomarse en cuenta algunas limitaciones derivadas del propio sistema de registro epidemiológico institucional, el cual, hasta el momento, no permite distinguir con precisión qué sustancias del grupo de opioides sintéticos en particular son las que han contribuido al incrementar en 300\% de la proporción de usuarios de estas drogas durante el periodo de 2013 a 2019.

\section{REFERENCIAS}

Baldwin, N., Gray, R., Goel, A., Wood, E., Buxton, J. A., \& Rieb, L. M. (2018). Fentanyl and heroin contained in seized illicit drugs and overdose-related deaths in British Columbia, Canada: an observational analysis. Drug and Alcohol Dependence, 185, 322-327. doi: 10.1016/j.drugalcdep.2017.12.032

Berbesi, D., Segura-Cardona, A., Montoya-Vélez, L., \& Mateu-Gelabert, P. (2013). Consumo de heroína inyectada en Colombia y comportamientos de riesgo. Salud Mental, 36(1), 27-31. doi: 10.17711/SM.0185-3325.2013.004

Berbesi-Fernández, D. Y., Segura-Cardona, A., Montoya-Velez, L. P., \& Ramirez Medina, A. F. (2016). Factores asociados al consumo de cocaína en usuarios de heroína inyectable en Colombia. Salud Mental, 39(4), 205-211. doi: 10.17711/SM.0185-3325.2016.024 Brown, R. (2004). Heroin dependence. WMJ-MADISON, 103(4), 20-26. Cravioto, P., Medina-Mora, M. E., de la Rosa, B., Galván, F., \& Tapia-Conyer, R. (2003). Patrones de consumo de heroína en una cárcel de la frontera norte de México: barreras de acceso a tratamiento. Salud Pública de México, 45(3), 181-190.

De Molina, V. R., Llorens-Aleixandre, N., Bravo-Portela, M. J., Barrio-Anta, G., Valderrama, J. C., \& Sánchez-Mañez, A. (2007). Encuesta a personas admitidas a tratamiento por abuso o dependencia de heroína o cocaína, 2003-2004. Trastornos Adictivos, 9(4), 247-259. doi: 10.1016/S1575-0973(07)75654-4

Forsyth, C. J., Biggar, R. W., Chen, J., \& Burstein, K. (2017). Examining heroin use and prescription opioid misuse among adolescents. Criminal Justice Studies, 30(3), 320-329. doi: 10.1080/1478601X.2017.1286836

Gutiérrez, A. D. (2013). Consumo de drogas en pacientes de primer ingreso a tratamiento a Centros de Integración Juvenil. Enero Junio, 2013. (Informe de investigación 13-09a). México: Centros de Integración Juvenil. Recuperado de http://www.cij.gob.mx/ 
programas/Investigacion/Epidemiologica.asp

Gutiérrez, A. D. (2019). Consumo de drogas en solicitantes de tratamiento en Centros de Integración Juvenil. Drogas ilícitas. Primer semestre de 2019. (Informe de investigación 19-04a). México: Centros de Integración Juvenil. Recuperado de http://www.cij. gob.mx/programas/Investigacion/Epidemiologica.asp

Gutiérrez, E., Sáiz, P. A., García, N., Fernández, P., González, M. P., Fernández, J. J. \& Bobes, J. (2001). Evolución de la gravedad de la adicción a los dos años de tratamiento en pacientes heroinómano. Adicciones, 13(1), 51-60.

Hosztafi, S. (2011). Heroin addiction. Acta Pharmaceutica Hungarica, 81(4), 173-183.

Instituto Nacional de Psiquiatría Ramón de la Fuente Muñiz, Comisión Nacional Contra las Adicciones, Secretaría de Salud. (2015). Encuesta Nacional de Consumo de Drogas en Estudiantes 2014: Reporte de Drogas. México: INPRFM.

Instituto Nacional de Psiquiatría Ramón de la Fuente Muñiz, Instituto Nacional de Salud Pública, Comisión Nacional contra las Adicciones, Secretaría de Salud. (2017). Encuesta Nacional de Consumo de Drogas, Alcohol y Tabaco 2016-2017: Reporte de Drogas. Ciudad de México. México: INPRFM.

Isaza, C., Suárez, P., Henao, J., \& González, M. (2010). Características demográficas y de consumo en pacientes adictos a heroína o derivados de la coca. Investigaciones Andina, 12(21), 24-34. doi: 10.33132/01248146.245

Jain, A., \& Fisher, C. E. (2018). Heroin and Other Illicit Opioids. The American Opioid Epidemic: From Patient Care to Public Health, 49.

Meacham, M.C., Rudolph, A. E., Strathdee, S. A., Rusch, M. L., Brouwer, K. C., Patterson, T.L., ... Roesch, S. C. (2015). Polydrug Use and HIV Risk Among People Who Inject Heroin in Ti- juana, Mexico: A Latent Class Analysis. Substance Use \& Misuse, 50(10), 1351-1359, DOI: 10.3109/10826084.2015.1013132

Observatorio Europeo de las Drogas y las Toxicomanías y Europol. (2016). Informe sobre los mercados de drogas en la UE: Visión de conjunto estratégica. Luxemburgo: Oficina de Publicaciones de la Unión Europea.

Oficina de las Naciones Unidas contra la Droga y el Delito (UNODC). (2019). Informe Mundial sobre las Drogas 2019. Publicación de las Naciones Unidas.

Parker, H., Egginton, R., \& Elson, N. (2001). Unreachable? The new young heroin users. In UK Drugs Unlimited (pp. 98-127). Palgrave Macmillan, London.

Rodríguez, O. G., Villa, R. S., Hermida, J. R. F., Crespo, J. L. C., Pérez, J. M. E., \& Díaz, S. A. H. (2005). Comparación de pacientes cocainómanos y heroinómanos en el EuropASI. Adicciones, 17(1), 33.

Romo-González, T., \& Larralde, C. (2013). ¿Cuáles y qué tan graves son los daños a la salud individual y a la sociedad causados por el consumo de drogas lícitas e ilícitas? Psychologia, 7(1), 67-80.

Sanvisens, A., Rivas, I., Faure, E., Muñoz, T., Rubio, M., Fuster, D., ... Muga, R. (2014). Características de los pacientes adictos a la heroína admitidos en un programa de tratamiento con metadona. Medicina Clínica, 142(2), 53-58. doi:10.1016/j.medcli.2012.10.023

Secretaría de Salud, Consejo Nacional contra las Adicciones, Instituto Nacional de Psiquiatría Ramón de la Fuente Muñiz, Instituto Nacional de Salud Pública. (2011). Encuesta Nacional de Adicciones 2011, México: INSP. Recuperado de: www.conadic. salud.gob.mx

Schilling, R. (2019, Feb 16). The Most Addictive Drugs. Recuperado de http://www.askdrray.com/the-most-addictive-drugs/ 